\title{
Neonatal Gardner Fibroma Leads to Detection of Familial Adenomatous Polyposis: Two Case Reports
}

\author{
Mattias Schäfer ${ }^{1}$ Martina Kadmon ${ }^{2}$ Wolfgang Schmidt ${ }^{3}$ Irmgard Treiber ${ }^{4}$ Ute Moog ${ }^{5}$ \\ Christian Sutter $^{5}$ Maximilian Stehr $^{1}$
}

${ }^{1}$ Department of Pediatric Surgery and Urology, Cnopfsche Kinderklinik, Nürnberg, Germany

2 Medicine and Health Sciences, Carl von Ossietzky Universität Oldenburg, Oldenburg, Germany

${ }^{3}$ Department of Pediatrics, Section Pediatric Oncology, Cnopfsche Kinderklinik, Nürnberg, Germany

${ }^{4}$ Department of General, Visceral, and Transplant Surgery,

Universitätsklinikum Heidelberg, Germany

${ }^{5}$ Institute of Human Genetics, Universitätsklinikum Heidelberg,

Germany

Eur J Pediatr Surg Rep 2016;4:17-21

\author{
Address for correspondence Mattias Schäfer, MD, Department of \\ Pediatric Surgery and Urology, Cnopfsche Kinderklinik, St. Johannis- \\ Mühlgasse 19, Nürnberg 90419, Germany \\ (e-mail: mattias.schaefer@email.de).
}

\begin{abstract}
Keywords

- Gardner fibroma

- FAP

- neonatology

- familial adenomatous polyposis

- premalignant lesion

Gardner fibromas (GFs) have only recently been described as poorly circumscribed tumor-like lesions, which are exceedingly rare in children. GFs are associated with APC gene mutations and therefore with familial adenomatous polyposis (FAP). So far there is only very limited literature on GF in the neonatal period. We present two children with GF diagnosed at birth and subsequent FAP with very different clinical courses. In one case, the disease led to extensive surgery of the thoracic wall and detection of FAP in the father with the need of immediate proctocolectomy. In the other patient (with a positive family history for FAP) the disease remained stable. Our cases indicate that the diagnosis of GF in the neonatal period requires the exclusion of FAP both in the child as well as the parents. Since the clinical behavior of GF cannot be predicted, continuous monitoring is mandatory. Depending on tumor site and growth, individual therapeutic options must be thoroughly considered. Surgical resection, if necessary, has to be inevitably total to prevent recurrence.
\end{abstract}

\section{Importance for a Pediatric Surgeon}

Gardner fibroma especially in the neonatal period are strong suspect of underlying FAP. Continuous follow-up is mandatory because of unpredictable growth behavior and early onset of intestinal polyps.

\section{Introduction}

Familial adenomatous polyposis (FAP) is a rare cause of colorectal cancer and is caused by a wide spectrum of mutations in the APC gene. ${ }^{1,2}$ FAP usually presents with a 100 to 1,000 of precancerous colonic polyps. Without colectomy, colon cancer is inevitable. While pathogenic received

October 20, 2015

accepted after revision

March 6, 2016

published online

May 13, 2016

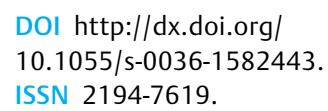

DOI http://dx.doi.org/

10.1055/s-0036-1582443.

ISSN 2194-7619.

(c) 2016 Georg Thieme Verlag KG Stuttgart . New York
License terms

(요 (1) $\Theta \circledast$ 
APC mutations have a complete penetrance in the colon, this is not the case in extracolonic manifestations. Gardner and Richards first described the association of FAP with a variety of extracolonic tumors, especially soft tissue lesions, but also osteomas, thyroid cancer, and hepatoblastoma. The triad of soft tissue lesions, osteomas, and dental abnormalities in FAP patients has therefore been named Gardner syndrome.,4 Gardner fibromas (GF) have been defined only recently as superficial and poorly circumscribed tumor-like lesions, which consist mainly of thick haphazardly arranged collagen bundles with few interspersed spindle cells of fibroblast type. ${ }^{5-7}$ GF are regarded as a precursor lesion of desmoid tumors which have a more cellular appearance. Both are associated with FAP (although it is unknown whether this is always the case).$^{8,9}$ The most common sites are the back, paraspinal region, and chest wall, but GF may occur in any part of the body. GF may precede the development of colonic adenomas and therefore lead to an early detection of FAP in otherwise asymptomatic patients. Although it is most frequently observed in the first decade of life, with $78 \%$ of the cases being diagnosed before 10 years of age, ${ }^{9}$ neonatal GF has only been reported in a single case report so far. ${ }^{10}$ In our report, we relate the cases of two patients with neonatal GF and very different findings.

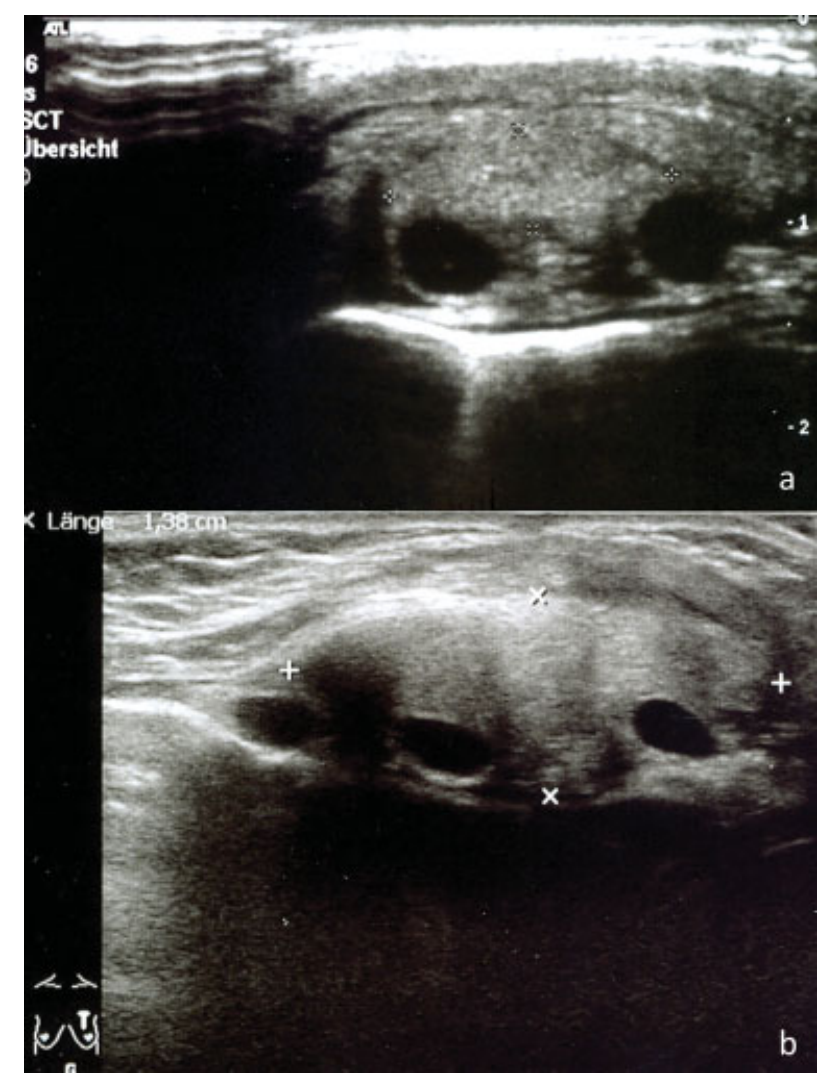

Fig. 1 Progression of the tumor of patient A. (a) Before first surgery. (b) Growing tumor size 5 months after first resection: $4.8 \times 3.5 \times 1.4 \mathrm{~cm}$.

\section{Patient Information}

\section{Patient A}

A 7-week-old otherwise healthy boy of nonconsangineous parents presented with a subcutaneous tumor on the left parasternal chest wall immediately after birth. Ultrasound was performed and showed a dense structure lying above rib level measuring $1.4 \times 0.5 \mathrm{~cm}$ ( $=$ Fig. $1 \mathrm{a}$ ). A resection was performed. Intraoperatively, only subtotal excision was possible due to poor circumscription. Histologically, a GF was confirmed ( - Fig. 2). Analysis of the family history revealed no family members with adenomas, fibromas, or colorectal cancer. The clinical and sonographic course showed progression of the fibroma after resection (-Fig. 1b). Magnetic resonance imaging (MRI) scans at the age of 5.5 and 9 months revealed a tumor size of $4.5 \times 4.0 \times 1.2$ and $6.2 \times 5.6 \times 1.8 \mathrm{~cm}$, respectively. Furthermore, the tumor had infiltrated the intercostal space and distal sternum at 9 months, eroding the bone, and was lying adjacent to the pericardium (-Fig. 3a). A radical resection was performed with partial resection of the distal sternum, ribs 7 to 9 on the left side and the medial part of the major pectoralis muscle. Closure of the chest wall was possible with insertion of a $5 \times 4 \mathrm{~cm}$ GoreTex®patch (W. L. Gore \& Associates, Inc., Medical Products Division, Flagstaff, Arizona, United States). Mobilization of major and minor pectoralis muscle as well as the abdominal wall muscles allowed full muscular covering and subsequent primary skin closure. Wound healing was uneventful and discharge was possible after 9 days. Histology confirmed progression of the GF. Resection margins were free of tumor except at the inferior end (cartilaginous part of rib 10). Regular MRI scans every 6 months during follow-up showed no recurrence (-Fig. 3b). Follow-up will be continued.

Molecular genetic analysis of the APC gene by polymerase chain reaction (PCR) and subsequent Sanger sequence analysis of all coding exons did not show any causative mutation. In addition, multiplex ligation-dependent probe amplification (MLPA, Kit P043, MRC-Holland, The Netherlands) analysis, which allows for the detection of potential deletions and duplications, showed a large heterozygous deletion affecting most of the APC coding region (exons 4-15 [GenBank NM_000038.3], equals exons 7-18 [GenBank NM_000038.5]) up to the most distal part of the gene (fragment W) (-Fig. 4). The deletion was confirmed on a second blood sample and classified as a bona fide pathogenic alteration because of its large size.

Subsequent testing of the parents showed the same APC deletion in the father who had no complaints. After having established the diagnosis he underwent colonoscopy, which led to the diagnosis of cancer of the sigmoid colon. The patient underwent restorative proctocolectomy (R0) and ileal pouch-anal anastomosis, the histological workup verified a pT3, pN2 (11/52), L1, G2 adenocarcinoma. So far, he has developed no desmoids.

The paternal grandmother did not show the familial APC deletion, the paternal grandfather was unavailable for investigation. 


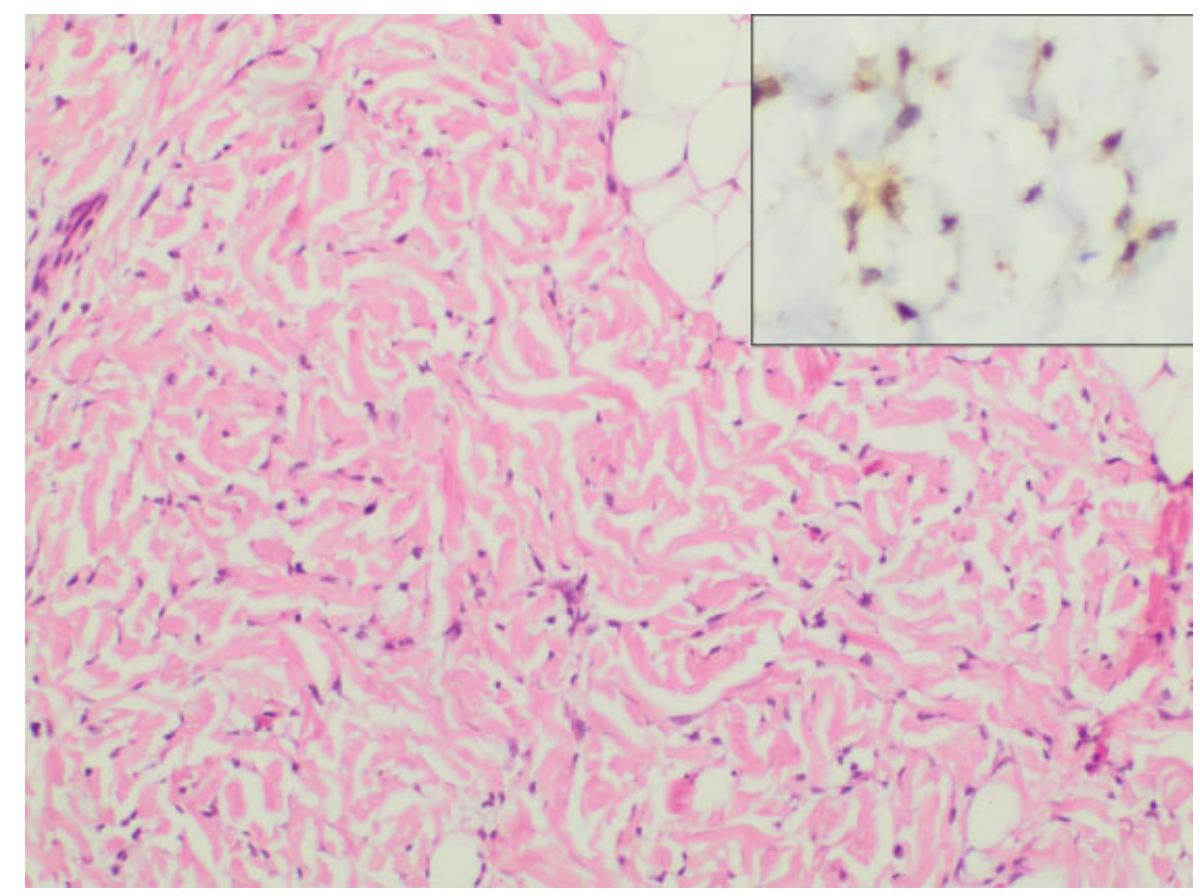

Fig. 2 Histological images of the tumor. H\&E stain $(\times 40)$ shows thick haphazardly arranged collagen bundles with few interspersed spindle cells of fibroblast type. Insert shows positive immunohistochemistry staining of $\beta$-catenin in the tumor $(\times 100)$. H\&E, Hematoxylin and eosin. (Image courtesy of A. Kaiser, MD, Klinikum Nürnberg, Institute of Pathology).

\section{Patient B}

The otherwise healthy boy of nonconsangineous parents was diagnosed with a left-sided paravertebral soft tissue tumor measuring $6 \times 3 \times 1 \mathrm{~cm}$ at birth. Due to known FAP
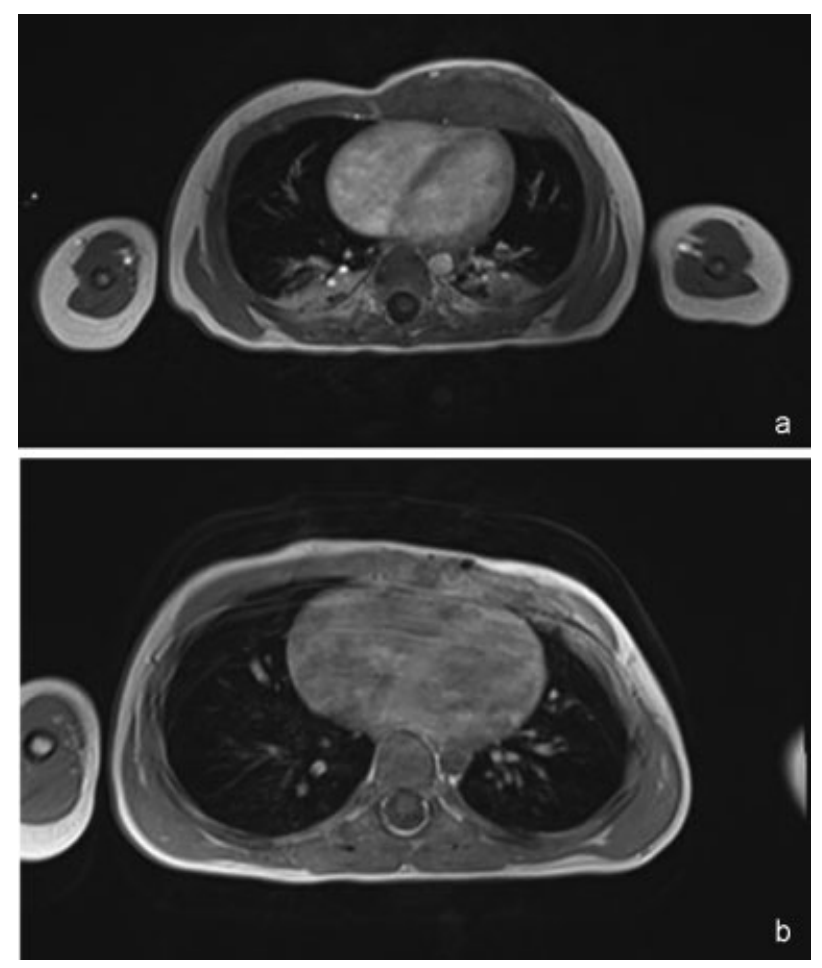

Fig. 3 MRI scans of patient A. (a) Tumor at the age of 9 months. (b) Tumor site 24 months postoperatively without recurrence. MRI, magnetic resonance imaging. in the father the soft tissue tumor was assumed to be an extracolonic manifestation of FAP.

Because of tumor progression during the next 4 months ( - Fig. 5) a biopsy was performed under general anesthesia. Histology confirmed GF consisting of fatty tissue interspersed with thick collagen bundles, many small blood vessels and some dispersed mast cells. Other cells and especially fibroblasts were rare.

Subsequent MRI scans showed no significant further tumor growth. Meanwhile the boy is 17 years old. He developed osteomas of the skull and intestinal polyps were confirmed by colonoscopy but without the necessity for surgical intervention so far.

The father of the boy had developed clinical symptoms of FAP at the age of 27 and underwent prophylactic restorative proctocolectomy and ileal pouch-anal anastomosis at the age of 28. Within 3 years after surgery he developed severe retroperitoneal desmoid disease leading to hydronephrosis necessitating nephroureterectomy. After 6 months he underwent a further desmoid tumor resection in the abdominal wall, the attempted resection of a large pelvic desmoid failed due to poor circumscription.

The paternal grandparents were both healthy without signs of FAP.

Molecular genetic analysis by PCR and Sanger sequence analysis of all coding exons showed a heterozygous frameshift mutation c.4393_4394delAG, p.Ser1465Trpfs*3 in exon 15, segment $H$ (equals exon 18, segment $H$ [GenBank NM_000038.5]) of the APC gene leading to introduction of a stop codon at amino acid position 1467, thereby causing a truncated nonfunctional or functionally impaired APC protein. 


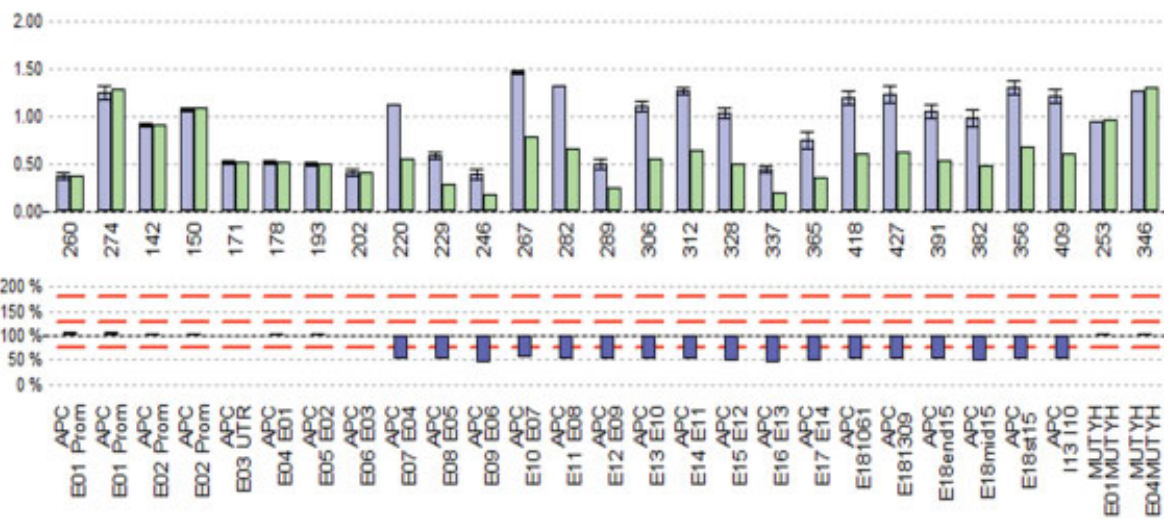

Fig. 4 Deletion/duplication analysis using multiplex ligation-dependent probe amplification showing monoallelic deletion affecting most of the APC coding region, showing that the gene dose of exon 4 onwards is reduced by $50 \%$ and proving the heterozygous deletion of a large portion of $A P C$.

\section{Discussion}

Literature regarding the clinical significance of neonatal onset of GF is very sparse. Whereas early childhood GF have been
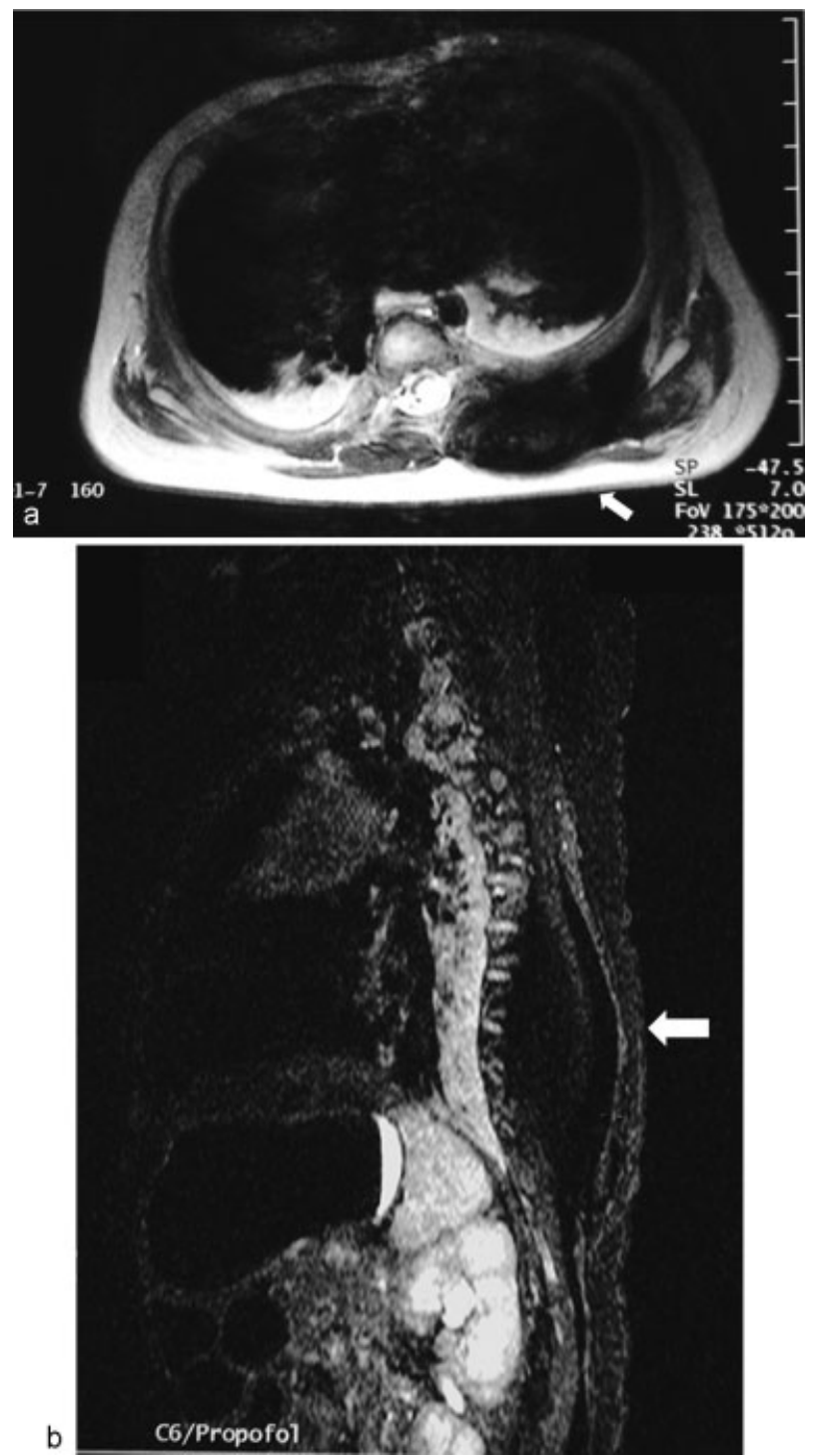

Fig. 5 MRI scan 16 weeks after birth Tumor size: $8 \times 5 \mathrm{~cm}$. (a) Sagittal view. (b) Axial view. Arrows indicate tumor location. reported and the assumption has been made that there is a strong connection to $\mathrm{FAP},{ }^{9}$ to our knowledge there is only one report of a neonatal onset of GF leading to the diagnosis of FAP in an otherwise asymptomatic child. ${ }^{10}$ In this report the patient's parents were healthy and had negative genetic testing, so that a de novo mutation is most probable. In contrast, in our patient $\mathrm{A}$, testing of the parents revealed the same mutation in the father and the diagnosis of abundant intestinal polyposis and sigmoid cancer in colonoscopy. This is to our knowledge the first report of a neonatal GF requiring immediate total proctocolectomy in a parent.

Coffin et al noted that $29 \%$ of all patients with GF developed this lesion under the age of 1 year, with an onset at 2 months of age. ${ }^{9}$ It is possible that in this series some GF may have existed unnoticed since birth. Although the overall (and possibly underestimated) association rate with FAP is as high as $69 \%$ in this study, no information is available about relevance of GF as a sentinel lesion for FAP in neonatal patients.

Viera et al reported two infants with GF which led to previously unrecognized FAP in the families. ${ }^{11}$ In their patients, immunofluorescent staining of $\beta$-catenin in the tumor, which accumulates in cells with biallelic truncation of APC because of the impairment of APC-mediated degradation of $\beta$ catenin, ${ }^{4}$ led to the strong suspicion of underlying FAP. This may well be a future diagnostic tool in evaluating the role of GF in detection of FAP. ${ }^{12}$

Of note, in our patient $A$ the tumor showed a rapid progression which necessitated extensive surgery, while the lesion remained stable in patient $B$. Although the invasive rapid growth is already a sign of a desmoid tumor, it was histologically still regarded as a GF.

It may be discussed if site and type of APC mutations may play a role in disease outcome, since patient A carries a large deletion encompassing most of the APC coding region, whereas patient $B$ has an underlying mutation in the desmoid region of the APC gene. ${ }^{4}$ Screening for somatic APC mutations in the tumor indicating loss of heterozygosity might have been instructive. The loss of the wildtype allele of APC in the tumor has been shown to be a relatively frequent event in desmoid tumors in adult FAP individuals. ${ }^{13}$ Unfortunately, these data are not available in our patients. 
However, it is known that trauma or surgery can be a predisposing factor to desmoid development in FAP. ${ }^{9}$ Therefore, the possibility that the first surgery triggered the intensive growth of the GF in patient A cannot be excluded. If surgery is performed, a total excision of GF with a wide margin should be aimed for, but can be technically demanding or even impossible because of the poor circumscription or anatomic boundaries. In general, prediction of GF behavior is impossible. For desmoid tumors it has been stated that approximately 5 to $10 \%$ appear to resolve spontaneously, $30 \%$ undergo cycles of progression and resolution, $50 \%$ remain stable after diagnosis, but $10 \%$ tend to progress rapidly, growing to massive sizes and infiltrating adjacent tissue. ${ }^{4}$ It is also known that there is a relationship between frequency of extracolonic manifestations and the position of APC germline mutations in patients with FAP. ${ }^{4,14,15}$ For example, the risk of developing desmoid tumors is increased in patients with an APC mutation 3' to codon $1399,{ }^{16}$ and reaches up to $100 \%$ in patients with a mutation in codons 1395 to $1493 .^{17}$ Studies correlating the risk of developing GF with a particular genotype are not available.

Nevertheless, surgery is the first-line therapy in fastgrowing GF. In older patients with advanced cases with progression into desmoid tumor cytotoxic chemotherapy may be discussed, but was not considered promising in our case because of the young age and the GF still being considered a benign entity. ${ }^{18,19}$

Continuous follow-up is mandatory, since early onset of intestinal polyps in children from the age of 4 to 6 years has been reported. ${ }^{20-22}$ In general, routine colonoscopies have been suggested to start at the age of 10 . Because of the unusual early clinical onset of intestinal symptoms (bloody stools and $>75$ polyps in colonoscopy) at the age of 4 years in a previously reported patient with neonatal GF, we recommend early annual routine colonoscopy in patient A starting from the age of 2 years. ${ }^{10}$

Since approximately $25 \%$ of FAP cases are caused by de novo mutations, the development of a GF in a child must raise suspicion of an underlying FAP even if the family history is negative. Performing a sound genetic testing for FAP including sequencing of the complete coding region of the $A P C$ gene and quantitative analysis by, for example, MLPA in every child with GF is strongly advised.

\section{References}

1 Half E, Bercovich D, Rozen P. Familial adenomatous polyposis. Orphanet J Rare Dis 2009;4:22

2 Fearnhead NS, Britton MP, Bodmer WF. The ABC of APC. Hum Mol Genet 2001;10(7):721-733

3 Gardner EJ, Richards RC. Multiple cutaneous and subcutaneous lesions occurring simultaneously with hereditary polyposis and osteomatosis. Am J Hum Genet 1953;5(2):139-147

4 Groen EJ, Roos A, Muntinghe FL, et al. Extra-intestinal manifestations of familial adenomatous polyposis. Ann Surg Oncol 2008; 15(9):2439-2450

5 Wehrli BM, Weiss SW, Yandow S, Coffin CM. Gardner-associated fibromas (GAF) in young patients: a distinct fibrous lesion that identifies unsuspected Gardner syndrome and risk for fibromatosis. Am J Surg Pathol 2001;25(5):645-651

6 Clark SK, Smith TG, Katz DE, Reznek RH, Phillips RK. Identification and progression of a desmoid precursor lesion in patients with familial adenomatous polyposis. Br J Surg 1998;85(7): 970-973

7 Coffin CM. Gardner fibroma. In: Fletcher CDM, Unni KK, Mertens F, eds. Pathology and Genetics: Tumours of Soft Tissue and Bone. World Health Organization Classification of Tumours. Lyons, France: IARC Press; 2002. https://www.iarc.fr/en/publications/ pdfs-online/pat-gen/bb5/BB5.pdf

8 Cates JM, Stricker TP, Sturgeon D, Coffin CM. Desmoid-type fibromatosis-associated Gardner fibromas: prevalence and impact on local recurrence. Cancer Lett 2014;353(2):176-181

9 Coffin CM, Hornick JL, Zhou H, Fletcher CD. Gardner fibroma: a clinicopathologic and immunohistochemical analysis of 45 patients with 57 fibromas. Am J Surg Pathol 2007;31(3): 410-416

10 Levesque S, Ahmed N, Nguyen VH, et al. Neonatal Gardner fibroma: a sentinel presentation of severe familial adenomatous polyposis. Pediatrics 2010;126(6):e1599-e1602

11 Vieira J, Pinto C, Afonso M, et al. Identification of previously unrecognized FAP in children with Gardner fibroma. Eur J Hum Genet 2015;23(5):715-718

12 Kattentidt Mouravieva AA, Geurts-Giele IR, de Krijger RR, et al. Identification of Familial Adenomatous Polyposis carriers among children with desmoid tumours. Eur J Cancer 2012;48(12): 1867-1874

13 Robanus-Maandag E, Bosch C, Amini-Nik S, et al. Familial adenomatous polyposis-associated desmoids display significantly more genetic changes than sporadic desmoids. PLoS ONE 2011;6(9): e24354

14 Enomoto M, Konishi M, Iwama T, Utsunomiya J, Sugihara KI, Miyaki M. The relationship between frequencies of extracolonic manifestations and the position of APC germline mutation in patients with familial adenomatous polyposis. Jpn J Clin Oncol 2000;30(2):82-88

15 Bertario L, Russo A, Sala P, et al; Hereditary Colorectal Tumor Registry. Multiple approach to the exploration of genotype-phenotype correlations in familial adenomatous polyposis. J Clin Oncol 2003;21(9):1698-1707

16 Sinha A, Tekkis PP, Neale KF, Phillips RK, Clark SK. Risk factors predicting intra-abdominal desmoids in familial adenomatous polyposis: a single centre experience. Tech Coloproctol 2010; 14(2):141-146

17 Wallis YL, Morton DG, McKeown CM, Macdonald F. Molecular analysis of the APC gene in 205 families: extended genotypephenotype correlations in FAP and evidence for the role of APC amino acid changes in colorectal cancer predisposition. J Med Genet 1999;36(1):14-20

18 Kitamura A, Kanagawa T, Yamada S, Kawai T. Effective chemotherapy for abdominal desmoid tumor in a patient with Gardner's syndrome. Report of a case. Dis Colon Rectum 1991;34(9):822-826

19 Bhama PK, Chugh R, Baker LH, Doherty GM. Gardner's syndrome in a 40-year-old woman: successful treatment of locally aggressive desmoid tumors with cytotoxic chemotherapy. World J Surg Oncol 2006;4:96

20 Attard TM, Tajouri T, Peterson KD, Tinley S, Thorson AG, Lynch HT. Familial adenomatous polyposis in children younger than age ten years: a multidisciplinary clinic experience. Dis Colon Rectum 2008;51(2):207-212

21 Distante S, Nasioulas S, Somers GR, et al. Familial adenomatous polyposis in a 5 year old child: a clinical, pathological, and molecular genetic study. J Med Genet 1996;33(2):157-160

22 Caspari R, Friedl W, Mandl M, et al. Familial adenomatous polyposis: mutation at codon 1309 and early onset of colon cancer. Lancet 1994;343(8898):629-632 